\title{
ISOTONE FUNCTIONS ON PARTIALLY ORDERED LINEAR ALGEBRAS WITH A MULTIPLICATIVE DIAGONAL MAP
}

\author{
TAEN-YU DAI AND RALPH DEMARR ${ }^{1}$
}

\begin{abstract}
The diagonal of the product of two triangular matrices is the product of the diagonals of each matrix. This idea is used to characterize Dedekind $\sigma$-complete lattice ordered linear algebras which admit isotone functions with familiar functional and order properties as possessed by the real-valued logarithm or root function.
\end{abstract}

1. Motivated by the work of Kadison and Singer [5] on triangular operator algebras, the authors [3] characterized abstract partially ordered linear algebras (polas) which have the order properties similar to an algebra of real upper triangular matrices. The main idea of the characterization we used in [3] is that in an algebra of upper triangular matrices the diagonal of the product of two matrices is equal to the product of the diagonals. We use the same idea in this paper to characterize the polas which admit an isotone function with familiar functional and order properties as possessed by the real-valued logarithm function or root function on the real line.

A dsc-pola, denoted by $A$, is a real linear associative algebra which satisifes the following two conditions: (1) It is partially ordered so that it is a directed partially ordered linear space and $0 \leqslant x y$ whenever $0 \leqslant x, y \in A$. (2) It is Dedekind $\sigma$-complete, i.e., if $x_{n} \in A, x_{1} \geqslant x_{2}>\cdots>0$, then $\inf \left\{x_{n}\right\}$ exists. Order convergence can be defined as usual. In this paper, we assume $A$ has a multiplicative identity $1 \geqslant 0$. Let $I=\{y \in A: 1 \leqslant y$ and $\left.y^{-1}>0\right\}$. Define $A_{1}=I-I$. It is shown in [2] that $A_{1}$ is an order-closed and order-convex commutative subalgebra of $A$ which behaves much like an algebra of real-valued functions. $A_{1}$ is called the diagonal or functional part of $A$. For detailed discussions and examples of $A_{1}$, see [2]; there we used the term polac instead of dsc-pola. A general discussion of lattices and lattices of operators may be found in [1] and [7].

Next we define a diagonal projection (DP) map $\Delta: A \rightarrow A_{1}$ to be a linear map such that $\Delta(1)=1$ and if $x \geqslant 0$, then $x \geqslant \Delta(x) \geqslant 0$. Since $\Delta$ is linear, it follows trivially that $\Delta$ is isotone, i.e., $\Delta(y)>\Delta(z)$ whenever $y>z, y, z \in$ $A$. This map was studied in [3]. Example 2.4 in [3] shows that in some dsc-pola there may not exist a $D P$ map; however, if it exists, it is unique [3,

Received by the editors May 13, 1976 and, in revised form, September 24, 1976.

AMS (MOS) subject classifications (1970). Primary 06A70; Secondary 47B55.

Key words and phrases. Dedekind $\sigma$-complete partially ordered linear algebra, lattice, diagonal, triangular matrices, matrix inequality, isotone functions.

${ }^{1}$ The authors wish to thank the referee for his valuable suggestions. 
Theorem 3.3]. But, when $A$ is a lattice, there always exists a $D P$ map [2, p. 671]. For convenience, we state the construction briefly. For any positive $x \in A$, define $\Delta(x)=\sup \{x \wedge n 1\}$. Since $0 \leqslant x \wedge n 1 \leqslant n 1$, as well as $x \wedge$ $n 1<x$, and since $A_{1}$ is order-convex and order-closed, we have $x \wedge n 1 \in A_{1}$ and $\Delta(x) \in A_{1}$. For any $y \in A$, define $\Delta(y)=\Delta\left(y^{+}\right)-\Delta\left(y^{-}\right) \cdot\left(y^{+}=y \vee\right.$ $0, y^{-}=(-y) \vee 0$.) Then $\Delta(y) \in A_{1}$. This map $\Delta$ is a diagonal projection map. (In [2, p. 671], notation $d(\cdot)$, rather than $\Delta(\cdot)$, was used.) Also, the reader should note that the concept of a $D P$ map is related to the so-called "diagonal process" defined by Kadison and Singer; see [4, p. 387].

Examples of a DP map will be given in $\$ 2$. We now state some important basic properties of a $D P$ map.

THEOREM 1.1. Suppose a DP map $\Delta$ exists on $A$. Then

(i) if $a \in A_{1}$, then $\Delta(a)=a$;

(ii) if $a \in A_{1}$, then $\Delta(a x)=\Delta(x a)=a \Delta(x)$ for $x \in A$; and

(iii) if $x, y \in A, 0 \leqslant x, 0 \leqslant y$, then $0 \leqslant \Delta(x) \Delta(y) \leqslant \Delta(x y)$.

Proof. See [3, Theorem 3.2].

Example 2.1 in the following section clearly indicates that (iii) is, in general, a strict inequality. In case that $\Delta(x y)=\Delta(x) \Delta(y)$ for all $x, y \in A$, we say $\Delta$ is multiplicative. In $\S 3$, we shall show that the admittance of some special kind of isotone functions on $A$ implies $\Delta$ is multiplicative.

2. Some elementary examples will be given to illustrate the basic concepts involved in this paper. For more examples of a diagonal projection map, we refer to $[3, \S 2]$.

EXAMPLE 2.1. Let $A$ be the algebra of all the real $n$-by- $n$ matrices with entry by entry ordering. Then $A$ is a dsc-pola (also a lattice). $A_{1}$ is nothing but all the diagonal matrices of $A$. For $y=\left[\alpha_{i j}\right] \in A$, define $\Delta(y)$ to be the diagonal part of $y$. It is trivial to verify that $\Delta$ is the $D P$ map, but $\Delta$ is not multiplicative.

EXAMPLE 2.2. Let $A$ be the algebra of all the real $n$-by- $n$ upper triangular matrices with entry by entry ordering. Define $\Delta$ as in Example 2.1 above. The reader should note that $\Delta$ is a multiplicative $D P$ map in this example.

On certain class of dsc-polas, there always exists some isotone function having the familiar functional and order properties as possessed by the real-valued logarithm or root function on the line; see Remark 3.8. We describe those functions precisely as follows. Let $H=\{x \in A: 1 \leqslant x\}$. An isotone function $f: H \rightarrow A$ is said to have the logarithm property if it satisfies the following three conditions:

(1) $f(x y)=f(x)+f(y)$ for $x, y \in H$;

(2) $f(x)<x-1$; and

(3) there exists $a \in H$ such that $f(a) \geqslant 1$.

Similarly, an isotone function $g: H \rightarrow A$ is said to have the root property if meets the following three conditions:

(1) $g(x y)=g(x) g(y)$; 
(2) $g(x)-1 \leqslant \lambda(x-1)$ for a fixed $\lambda, 1>\lambda>0$;

(3) there exists $a \in H$ such that $1 \leqslant g(a)-1$.

Note that (2) in the definition of $f$ (resp. $g$ ) is a standard inequality for the usual logarithm (resp. root) function. Without (3) we could get a trivial $f$ (resp. $g$ ) by defining $f(x)=0$ (resp. $g(x)=0$ or $g(x)=1$ ) for all $x \in H$.

EXAMPLE 2.3. Let $A$ be as in Example 2.2 above with $n=2$. For $1 \leqslant x=$ $\left(\begin{array}{ll}\boldsymbol{\alpha} & \boldsymbol{\nu} \\ 0 & \beta\end{array}\right)$, define

$$
f(x)=\left(\begin{array}{cc}
\log _{3} \alpha & 0 \\
0 & \log _{4} \beta
\end{array}\right) .
$$

Then we can check easily that $f$ is a function with the logarithm property.

ExAmple 2.4. Let $A$ be as in Example 2.3. For $1 \leqslant x=\left(\begin{array}{ll}\alpha & \nu \\ 0 & \beta\end{array}\right)$, define

$$
g(x)=\left(\begin{array}{cc}
\alpha^{1 / 2} & 0 \\
0 & \beta^{1 / 3}
\end{array}\right) .
$$

Choose $\lambda=1 / 2$. Again, it is easy to check that $g$ is a function with the root property.

3. In [3], various algebraic (order) conditions on $A$ were given which imply that a diagonal projection map is multiplicative. In this paper, we first prove the following theorem.

THEOREM I. Suppose a lattice dsc-pola $A$ admits a function $f$ with the logarithm property. Then the diagonal projection map $\Delta$ is multiplicative.

The theorem will be proved with the aid of a series of lemmas. Observe $f(1)=f(1)+f(1)$ gives $f(1)=0$.

Lemma 3.1. If $x \in H$, then $f(x) \in A_{1}$.

Proof. By isotonicity and (1), (2) in the definition of $f$, we have

$$
\begin{aligned}
0 & =f(1) \leqslant f(x) \leqslant f(n 1+x)=f(n 1)+f\left(1+n^{-1} x\right) \\
& \leqslant(n-1) 1+\left(1+n^{-1} x-1\right)=(n-1) 1+n^{-1} x
\end{aligned}
$$

for all positive integers $n$.

Since $A$ is lattice, we may write $f(x)=x_{n}+w_{n}$, where $0 \leqslant x_{n} \leqslant(n-1) 1$ and $0 \leqslant w_{n} \leqslant n^{-1} x$ for all $n$. From the fact that $A_{1}$ is order-convex and order-closed, we have $x_{n} \in A_{1}$ and $f(x)=\lim _{n \rightarrow \infty} x_{n} \in A_{1}$.

LEMMA 3.2. $f(x)=f(\Delta(x))$ for $x \in H$.

Proof. Since $1 \leqslant x$ and $\Delta$ is a $D P$ map, we have $1 \leqslant \Delta(x) \leqslant x$. This, by the isotone property of $f$, implies $f(\Delta(x)) \leqslant f(x)$. Next, observe $\Delta(x) \in A_{1} \cap$ $H$. By the definition of $A_{1}$ and Lemma I.6.6(ii) of [2], we know $\Delta(x)^{-1}>0$. Thus if $y=\Delta(x)^{-1} x$, then $1 \leqslant y$ and $\Delta(y)=1$ (Theorem 1.1(ii)). From $x=\Delta(x) y$, it follows that $f(x)=f(\Delta(x))+f(y) \leqslant f(\Delta(x))+y+1$. Now let us apply $\Delta$ to both sides of this inequality. Since both $f(x)$ and $f(\Delta(x))$ belong to $A_{1}$, using Thoerem 1.1(i), we obtain $f(x) \leqslant f(\Delta(x))$. Therefore, $f(x)=f(\Delta(x))$. 
LEMMA 3.3. If $1 \leqslant z \in A_{1}$ and $f(z)=0$, then $z=1$.

Proof. Note that $f\left(z^{n}\right)=n f(z)=0$; and $1 \leqslant z \in A_{1}$ implies $0<z^{-n}$ for all positive integers $n$. Now by the definition of $f$, there exists $a \in H$ such that $f(a)>1$. Together, those facts show that

$$
1<f\left(a+z^{n}\right)=f\left(z^{n}\right)+f\left(1+z^{-n} a\right) \leqslant 1+z^{-n} a-1=z^{-n} a .
$$

Therefore, $1 \leqslant z^{n} \leqslant a$ for all $n$. This means $z=1$.

Proof of Theorem I. For $x, y \in H$, using Lemma 3.2 twice, we have

$$
\begin{aligned}
f(\Delta(x) \Delta(y)) & =f(\Delta(x))+f(\Delta(y))=f(x)+f(y) \\
& =f(x y)=f(\Delta(x y)) .
\end{aligned}
$$

Let $c=\Delta(x) \Delta(y), d=\Delta(x y)$. Then $1 \leqslant c \leqslant d \in A_{1}$ by Theorem 1.1(iii). Thus, $1 \leqslant c^{-1} d$. From the relation $f(d)=f(c)+f\left(c^{-1} d\right)$ and the above result, it follows that $f\left(c^{-1} d\right)=0$. By Lemma 3.3, $c^{-1} d=1$ or $c=d$, i.e., $\Delta(x y)=\Delta(x) \Delta(y)$ for $x, y \in H$. Since $A$ is directed and $\Delta$ is linear, it is straightforward to verify that $\Delta$ is multiplicative.

TheOREM II. Suppose a lattice dsc-pola $A$ admits a function $g$ with the root property. Then the diagonal projection map $\Delta$ is multiplicative.

Since the proof of this theorem is similar to the proof of the previous theorem, we only sketch the procedure.

LEMMA 3.4. $g(n 1) \in A_{1}$, and $\lim _{k \rightarrow \infty}\left[g\left(n_{k} 1\right) / n_{k}\right]=0$ for a subsequence $\left\{n_{k}\right\}$.

Proof. We first show $g(1)=1$. From the definition of $g$, it follows that $g(1)<1$ and $g(a)=g(a) g(1)>2(1)$. By [2, Theorem I.6.3], $g(1)$ has an inverse. This and the fact that $g(1)=g(1)^{2}$ yield $g(1)=1$. By isotonicity, we have $1=g(1) \leqslant g(n 1)<1+\lambda(n-1) 1$. Consequently, $g(n 1) \in A_{1}$. Let $k$ be an arbitrary positive integer. Then

$$
g(n 1)=(g(\sqrt[k]{n} 1))^{k} \leqslant(1+\lambda(\sqrt[k]{n}-1) 1)^{k} .
$$

Thus

$$
n^{-1} g(n 1)<\left((1-\lambda) n^{-1 / k} 1+\lambda 1\right)^{k}
$$

In particular, when $n=n_{k}=k^{k}$, we have

$$
0 \leqslant\left(n_{k}\right)^{-1} g\left(n_{k} 1\right) \leqslant\left((1-\lambda) k^{-1} 1+\lambda 1\right)^{k} \text {. }
$$

Finally, $1>\lambda>0$ implies $\lim _{k \rightarrow \infty}\left(n_{k}\right)^{-1} g\left(n_{k} 1\right)=0$.

Lemma 3.5. $g(x) \in A_{1}$ for $x \in H$.

Proof. Since

$$
\begin{aligned}
1 & <g(x)<g(x+n 1)=g(n 1) g\left(1+n^{-1} x\right) \\
& <g(n 1)\left(1+\lambda\left(1+n^{-1} x-1\right)\right)=g(n 1)+(\lambda / n) g(n 1) x
\end{aligned}
$$


we have from Lemma 3.4 that there exists a subsequence $\left\{n_{k}\right\}$ such that $1<g(x)<g\left(n_{k} 1\right)+\left(\lambda / n_{k}\right) g\left(n_{k} 1\right) x$ and $\lim _{k \rightarrow \infty}\left(n_{k}\right)^{-1} g\left(n_{k} 1\right)=0$. Since $A$ is a lattice, we can proceed as in Lemma 3.1 to conclude $g(x) \in A_{1}$.

LEMMA 3.6. $g(x)=g(\Delta(x))$ for $x \in H$.

Proof. Since $1 \leqslant \Delta(x) \leqslant x$, we have $g(\Delta(x)) \leqslant g(x)$. Let $\left.y=\Delta(x)^{-1} x\right\rangle$ 1. Then it follows as in the proof of Lemma 3.2 that $g(x)=g(\Delta(x))$.

Lemma 3.7. If $1 \leqslant z \in A_{1}$ and $g(z)=1$, then $z=1$.

Proof. Note that $g\left(z^{n}\right)=g(z)^{n}=1$. Then by the definition of $g$, we have

$$
1 \leqslant g\left(a+z^{n}\right)-1=g\left(z^{n}\right) g\left(1+z^{-n} a\right)-1<\lambda z^{-n} a,
$$

thus $1 \leqslant z^{n}<\lambda a$ for all $n$. This implies $z=1$.

The proof of Theorem II can be completed by noting that if we let $c=\Delta(x) \Delta(y), d=\Delta(x y)$ for $x, y \in H$, then it follows as in the proof of Theorem I that $g(c)=g(d)$. But, $1 \leqslant g(c) \in A_{1}$ and $g(d)=g(c) g\left(c^{-1} d\right)$ implies $g\left(c^{-1} d\right)=1$. Hence, by Lemma 3.7, $c=d$. Thus, $\Delta$ is multiplicative.

We now close the article by making the following comment.

REMARK 3.8. Any dsc-pola $A$ with a multiplicative $D P$ map $\Delta$ always admits a function with the logarithm (root) property which we explain as follows. In [6] it was proved that $A_{1}$ is algebraically and order isomorphic to an algebra $S$ of continuous, almost-finite, extended-real-valued functions defined on a compact Hausdorff space. Hence, we may identify $A_{1}$ with $S$. It is clear then for $1 \leqslant z \in A_{1}, \ln (z)$ and $\sqrt{z}$ also belong to $A_{1}$. For $1 \leqslant x \in$ $A$, we define $f(x)=\ln (\Delta(x))$ and $g(x)=\sqrt{\Delta(x)}$. It can be verified easily that $f$ and $g$ are functions with the logarithm property and the root property, respectively.

\section{REFERENCES}

1. G. Birkhoff, Lattice theory, Amer. Math. Soc. Colloq. Publ., vol. 25, 3rd ed., Amer. Math. Soc., Providence, R.I., 1967. MR 37 \#2638.

2. T. Y. Dai, On some special classes of partially ordered linear algebras, J. Math. Anal. Appl. 40 (1972), 649-682. MR 47 \#4890.

3. T. Y. Dai and R. DeMarr, Partially ordered linear algebras with multiplicative diagonal map, Trans. Amer. Math. Soc. 224 (1976), 179-187.

4. R. V. Kadison and I. M. Singer, Extensions of pure states, Amer. J. Math. 81 (1959), 383-400. MR 23 \# A1243.

5. _ Triangular operator algebras, Amer. J. Math. 82 (1960), 227-259. MR 22 \# 12409.

6. Richard Metzler, Representation of partially ordered linear algebras, Bull. Amer. Math. Soc. 80 (1974), 939-940. MR 50 \#6969.

7. H. H. Schaefer, Banach lattices and positive operators, Springer-Verlag, Berlin and New York, 1974.

Department of Mathematics, York College, City University of New York, Jamaica, NeW YORK 11451

Department of Mathematics, University of New Mexico, Albuquerque, New Mexico 